\title{
DIATOMÁCEAS CÊNTRICAS DA ZONA DE ARREBENTAÇÃO DE UMA ILHA AMAZÔNICA
}

\author{
Jislene Brito MATOS ${ }^{1}$ \\ Eliz Helena Nogueira CARDOSO ${ }^{1}$ \\ Luci Cajueiro Carneiro PEREIRA ${ }^{2}$ \\ Rauquírio Marinho da COSTA ${ }^{1, *}$
}

Recebido em: 08/03/2013

Aceito em: 07/06/2013

\section{RESUMO}

O presente trabalho visou determinar a composição das diatomáceas cêntricas (classe Coscinodiscophyceae) relacionando-a com as variações ambientais da praia da Princesa (Ilha de Algodoal), durante os meses de novembro de 2008, março, junho, setembro de 2009. As amostras biológicas foram coletadas com auxílio de uma rede de plâncton e posteriormente analisadas em laboratório. No presente estudo, a sazonalidade foi bem definida, com valores de salinidade e $\mathrm{pH}$ significativamente mais elevados durante o período seco, enquanto que a biomassa fitoplanctônica (clorofila a) apresentou as mais elevadas concentrações no mês chuvoso $\left(56,9 \pm 15,2 \mathrm{mg}^{-3}\right.$ - março), indicando condições eutróficas do ambiente. Foram registrados 39 táxons, distribuídos em 6 subclasses, 8 ordens e 13 famílias. O fitoplâncton esteve caracterizado por espécies marinhas planctônicas neríticas (52,4\%). Coscinodiscus perforatus Ehrenberg foi representativa no período chuvoso, enquanto que Dimeregramma minor (Gregory) Ralfs, durante o período seco. A sazonalidade local exerceu uma forte influência sobre as variáveis ambientais, principalmente sobre a salinidade, que aliada à ação dos ventos e correntes de maré, permitiram a contribuição de diferentes espécies em cada período sazonal.

Palavras chave: composição, microfitoplâncton, praia, Norte do Brasil.

\section{ABSTRACT}

This study aimed to determine the composition of centric diatoms (Class Coscinodiscophyceae) and to investigate the effect of some environmental variables on this community in "Princesa" Beach (Algodoal Island, Pará, Brazil) during the months of November 2008, and March, June and September 2009. Samples were collected with plankton nets and then analyzed in the laboratory. In the present study, seasonality was well defined showing values of salinity and $\mathrm{pH}$ significantly higher during the dry season, whereas phytoplankton biomass (chlorophyll a) showed the highest concentrations in the rainy month $\left(56.9 \pm 15.2 \mathrm{mg}^{-3} \mathrm{~m}^{-3}\right.$ - March) indicating eutrophic conditions of the environment. A total of 39 taxa were recorded and distributed in 6 subclasses, 8 orders and 13 families. Phytoplankton was characterized by neritic planktonic marine species (52.4\%). Coscinodiscus perforatus Ehrenberg was representative in the rainy season, and Dimeregramma minor (Gregory) Ralfs, during the dry season. Local seasonality exerted a strong influenced on the environmental variables, mainly salinity, which combined with the action of winds and tidal currents, allowed the contribution of different species in each of the seasonal periods studied.

Key words: composition, microphytoplankton, beach, Northern Brazil.

\section{INTRODUÇÃO}

A zona costeira amazônica brasileira apresenta uma riqueza significativa, em termos de recursos naturais, sendo caracterizada por diversos ambientes tais como, praias, manguezais, 
rios, estuários e florestas tropicais (KRAUSE; GLASER, 2003). As praias desta região são únicas e exuberantes, sendo consideradas ambientes de alta energia e influenciados por fortes ventos, altas taxas de precipitação e regime de macromarés semi-diurnas (MONTEIRO; PEREIRA; OLIVEIRA, 2009).

Dentre os grupos de organismos que habitam as zonas costeiras o fitoplâncton destaca-se por sua diversidade, apresentando até 13 divisões e mais de 10.000 espécies identificadas em águas costeiras e oceânicas (SURTHERS; RISSIK, 2008). Por seu caráter dinâmico, responde rapidamente às alterações físicas e químicas do meio aquático, sendo de extrema importância para a caracterização ecológica destes ambientes (VALIELA, 1995).

Estes organismos estão sujeitos a variações espaciais e temporais, condicionadas, principalmente, pela disponibilidade de luz, sais nutrientes, temperatura e salinidade (COSTA; MACEDO, 1987/1989; PHILIPS; BADYLARK; GROSSKOPF, 2002). Além disso, esta comunidade aquática também está sujeita a outros processos característicos de regiões costeiras, tais como, os ventos, ressurgências e a pluviometria (SASSI, 1991; REYNOLDS, 1992), sendo este último, o principal fator controlador da dinâmica sazonal do fitoplâncton nos ambientes costeiros tropicais e subtropicais (SOUSA et al., 2008; COSTA et al., 2011; MATOS et al., 2012).

Devido a sua abundância e diversidade, as diatomáceas constituem um dos principais grupos microfitoplânctônicos em ecossistemas amazônicos (SOUSA et al., 2008; MATOS et al., 2011). Este grupo se caracteriza por apresentar algas unicelulares ou coloniais providas de uma frústula silicosa e suas células apresentam clorofila a e $c$, carotenóides, fucoxantina, diatoxantina e diadinoxantina (MARGULIS; SCHWARTZ, 1998; BELL; HEMSLEY, 2000). As diatomáceas cêntricas possuem simetria radial, ausência de rafe, e apresentam numerosos plastídeos pequenos, dominando o plâncton costeiro (VAN DEN HOEK; MANNS; JAHNS, 1995).

Apesar de reconhecida a importância do fitoplâncton e das diatomáceas para regiões costeiras, ainda são poucos os estudos sobre esta comunidade em relação sua dinâmica, quando comparados com a extensa área costeira e diversificados ambientes aquáticos encontrados ao longo da costa Norte do Brasil. Estudos desta natureza na ilha de Algodoal, uma área de proteção ambiental localizada no município de Maracanã (nordeste do Pará) ainda são escassos (MATOS et al., 2012). São insipientes também as pesquisas sobre a dinâmica das diatomáceas cêntricas em ambientes costeiros do nordeste paraense (SANTANA; PAIVA; MELO, 2005). Portanto, o presente trabalho visou determinar o efeito das variáveis ambientais sobre a dinâmica temporal e biomassa das diatomáceas cêntricas nas águas da praia da Princesa, localizada na ilha de Algodoal, buscando ampliar os conhecimentos sobre estes organismos na região Norte do Brasil.

\section{ÁREA ESTUDADA}

A ilha de Algodoal está localizada no Município de Maracanã, correspondendo a uma área de aproximadamente $23 \mathrm{~km}^{2}$ (MASCARENHAS, 2006). Em novembro de 1990, a ilha de Algodoal foi declarada Área de Proteção Ambiental (APA), unidade de conservação de caráter insular, sob a Lei estadual no 5621 (BASTOS, 1996). Os habitantes dessa ilha são pessoas simples e receptivas que vivem, basicamente, da pesca, da agricultura de subsistência e atualmente do turismo.

A estação de coleta está localizada na porção norte da ilha, na denominada praia da Princesa (Fig.1), e assim como as demais praias desta ilha sofre a influência das marés e dos rios amazônicos, o que faz com que, nos períodos de baixa-mar, com o recuo da água, formem-se muitas lagoas naturais. A praia da Princesa possui, cerca de $14 \mathrm{~km}^{2}$ de extensão e é visitada por milhares de veranistas ao longo do ano. Nesta praia as alturas máximas de marés podem alcançar valores superiores a 5,8 m (marés equinociais de sizígia) e as correntes de maré possuem predominantemente direção SE-NW, na enchente e NW-SE, na vazante, com maiores intensidades e os maiores fluxos durante o período de enchente (SILVA et al., 2011).

Segundo a classificação climática de Köppen, o clima da região é do tipo Am (Tropical Úmido e Monções) caracterizado por ser muito úmido e megatérmico, com sazonalidade bem definida (MORAES et al., 2005). A precipitação média anual varia de $2.500 \mathrm{~mm}$ a $3.000 \mathrm{~mm}$, a 
temperatura média anual do ar está em torno de $27,7^{\circ} \mathrm{C}$. A umidade relativa do ar está em torno de $83 \%$, sendo os meses chuvosos os mais úmidos (MARTORANO et al. 1993).

\section{MATERIAL E MÉTODOS}

As coletas dos dados hidrológicos e das amostras fitoplanctônicas foram realizadas nos meses de novembro de 2008 (período seco), março (período chuvoso), junho (período chuvoso) e setembro de 2009 (período seco), na praia da Princesa (Ilha de Algodoal) em uma estação fixa (00 34' 40. 63" S 470 35' 02. 29" W) (Fig. 1B). As amostragens foram nictemerais efetuadas em marés de sizígia com intervalos de 6 horas, durante os regimes de baixa-mar e preamar.

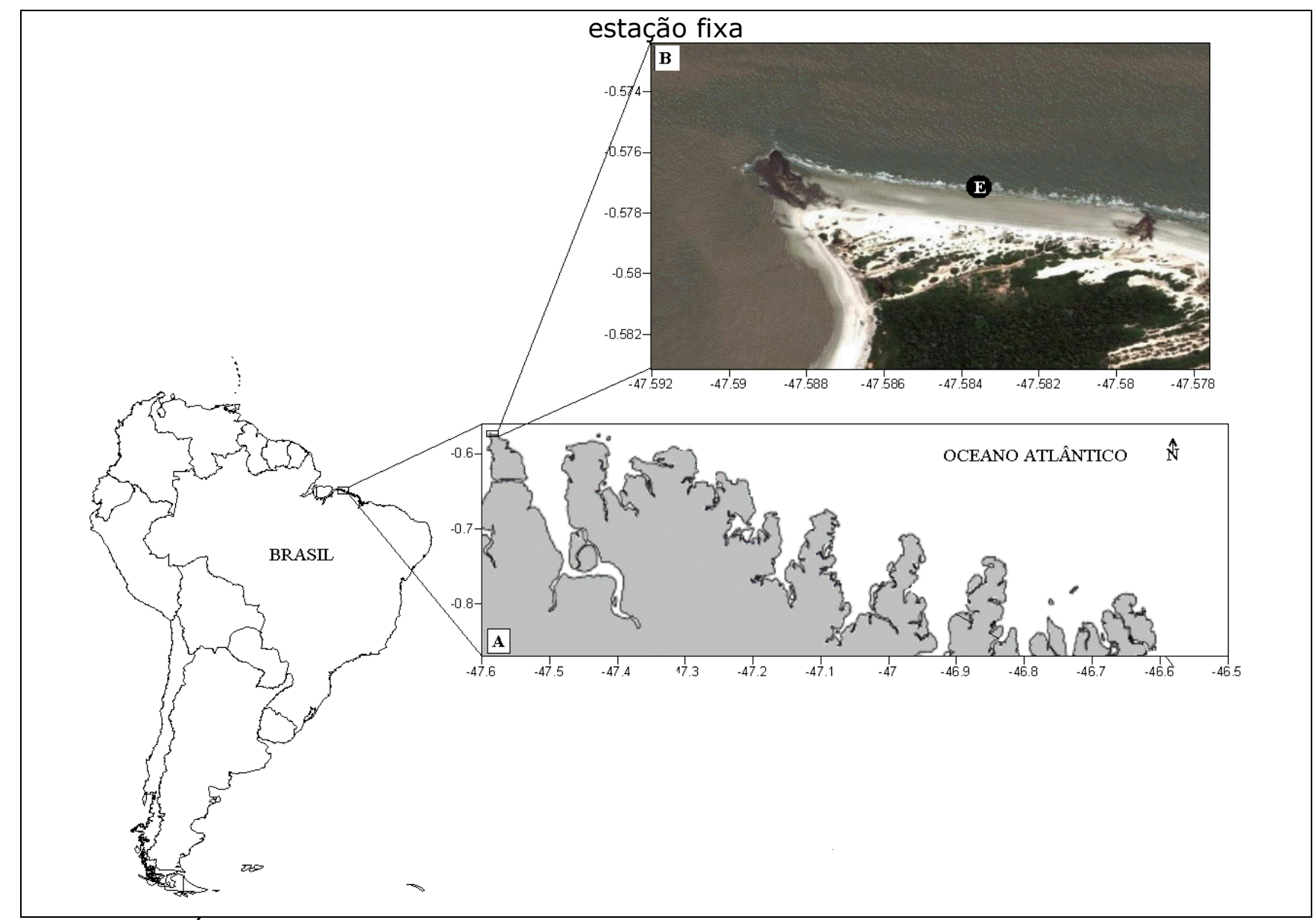

Figura 1 - Área de estudo situada no nordeste do Pará (A): praia da Princesa (B) com

localização da de coleta (E), Ilha de Algodoal, Pará, Brasil (Modificado de SOUSA et al., 2011).

A temperatura da água e salinidade foram medidas in situ com auxilio de um CTD (XR 420). Para as demais variáveis físico-químicas, bem como para determinação das concentrações de clorofila $a$, foram realizadas coletas subsuperficiais de água $(0-1 \mathrm{~m})$ com auxílio de uma garrafa de Niskin. As amostras destinadas a determinação das concentrações de oxigênio dissolvido foram fixadas com $1 \mathrm{ml}$ de sulfato de manganês e $1 \mathrm{ml}$ de iodeto alcalino e posteriormente transportadas para o laboratório. No laboratório, o pH foi mensurado com auxilio de um potenciômetro (pHmetro), a turbidez com um turbidímetro e as concentrações de oxigênio dissolvido foram medidas segundo o método de Winkler (STRICKLAND; PARSONS, 1968). As concentrações de clorofila a foram determinadas através do método espectrofotométrico, descrito por Parsons e Strickland (1963). 
As amostras para o estudo qualitativo do microfitoplâncton foram obtidas com auxílio de uma rede de plâncton $(64 \mu \mathrm{m})$, através da qual foram filtrados $400 \mathrm{~L}$ de água da camada subsuperficial da zona de arrebentação da praia. O material coletado foi fixado com formalina $4 \%$ neutralizada com tetraborato de sódio (Bórax). Para a análise qualitativa das diatomáceas cêntricas foram realizadas montagens lâmina-lamínula temporárias, sendo as mesmas observadas em microscópio binocular. Para cada amostra foram preparadas no mínimo cinco lâminas.

Os critérios de classificação de Round, Crawford e Mann (1990) foram empregados na elaboração da composição florística das diatomáceas cêntricas. Ainda para identificação das espécies foram utilizados os trabalhos de Silva-Cunha e Eskinazi-Leça (1990), Tomas (1997), Wood (1968), entre outros. O enquadramento ecológico das espécies foi realizado de acordo com Moreira Filho et al. (1990) e Valente-Moreira, Moreira Filho e Cunha (1994).

A frequência de ocorrência dos táxons foi calculada segundo Mateucci e Colma (1982) tendo sido estabelecidas as seguintes categorias: muito frequente $(\geq 75 \%)$, frequente $(<75 \%$ e $\geq$ $50 \%)$, pouco frequente $(<50 \%$ e $\geq 25 \%)$ e esporádica ( $<25 \%)$. Para abundância relativa foram utilizadas as categoria dominante para valores acima de $50 \%$ e abundante para os valores superiores a média (Lobo \& Leighton 1986).

Foram realizadas análises para determinar a normalidade e a homogeneidade das variâncias dos dados abióticos através dos testes de Lilliefors (CONOVER, 1971) e Bartlett"s (SOKAL; ROHLF, 1969), respectivamente, com auxílio do programa STATISTICA 6.0. Quando os dados não apresentaram distribuição normal, foi utilizada a transformação log $(x+1)$. Quando os dados foram normais e homogêneos foi aplicada ANOVA (fator único) para testar as possíveis diferenças nos parâmetros hidrológicos entre os diferentes meses, períodos sazonais e regimes de maré. No entanto, quando as variâncias não foram homogêneas, foi aplicado o teste não paramétrico de Mann-Whitney (U) (ZAR, 1999).

\section{RESULTADOS}

A salinidade média na praia da Princesa variou de 5,0 $\pm 0,0$ (baixa-mar de junho/08) a $35,5 \pm 0,2$ (preamar de novembro/08) (Fig. 2A), com valores significativamente mais elevados durante o período seco $(F=191,26 ; \mathrm{p}<0,05)$. Os valores médios de temperatura da água oscilaram entre $27,8 \pm 0,0^{\circ} \mathrm{C}$ (preamar de setembro/09) e 28,9 $\pm 0,6^{\circ} \mathrm{C}$ (baixa-mar de novembro/08) (Fig. 2A).

As concentrações de oxigênio dissolvido (OD) oscilaram, em média, entre 5,4 $\pm 1,6 \mathrm{mg} \cdot \mathrm{L}^{-1}$ (baixa-mar de março/09) e 8,1 $\pm 0,4 \mathrm{mg}^{-1} \mathrm{~L}^{-1}$ (preamar de setembro/09) (Fig. 2A), sendo observados valores significativamente mais elevados durante as preamares $(F=7,53 ; p<0,05)$. Quanto ao $\mathrm{pH}$, este manteve-se alcalino durante os meses de estudo, com médias variando de $7,0 \pm 0,1$ (baixa-mar de março/09) a 8,2 $\pm 0,0$ (preamar de novembro/08) (Fig. $2 \mathrm{~A}$ ), com valores significativamente mais elevados no período seco $(F=26,68 ; p<0,05)$.

Os valores médios da turbidez oscilaram entre $37,3 \pm 6,0$ UNT em novembro/08 e 106,3 \pm 45,7 UNT em setembro/09, sendo ambos os valores observados durante os regimes de preamar, com valores significativamente mais elevados durante o mês de setembro $(F=8,75$; $\mathrm{p}<0,05)$. As concentrações de clorofila a variaram, em média, de $4,6 \pm 2,3 \mathrm{mg} \cdot \mathrm{m}^{-3}$ no mês de junho/09 a 56,9 $\pm 15,2 \mathrm{mg}^{-3}$ no mês de março/09, sendo ambos os valores registrados durante a baixa-mar, com valores significativamente mais elevados no mês de março/09 $(U=0,0 ; p<0,05)$ (Fig. 3). 


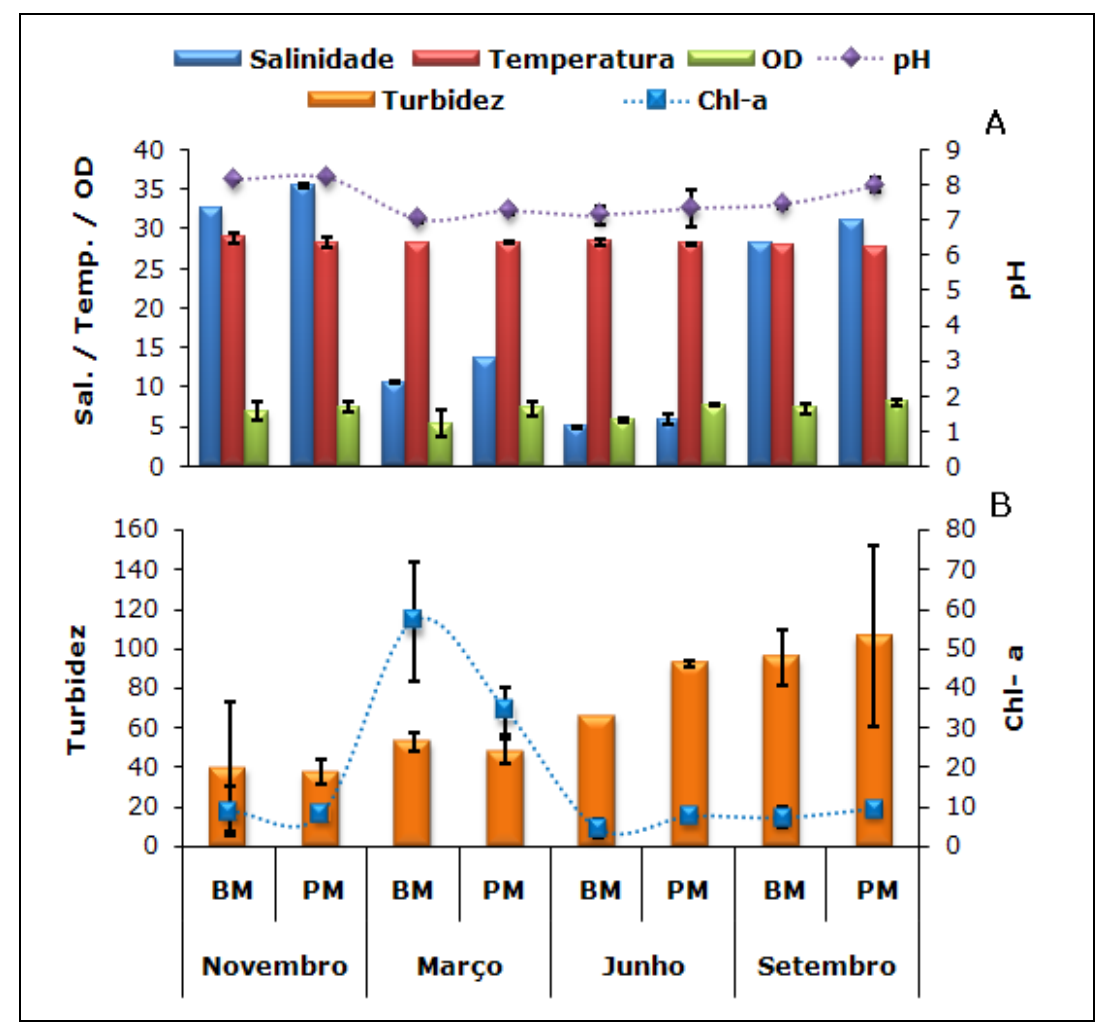

Figure 2 - Variação mensal ( $\pm D . P.):(A)$ salinidade, temperatura, $\mathrm{pH}$ e oxigênio dissolvido (OD) e (B) turbidez e clorofila a (Chl-a), na Praia da Princesa.

$\mathrm{Na}$ Ilha de Algodoal, a classe Coscinodiscophyceae (diatomáceas cêntricas) esteve representada por 39 táxons, distribuídos em 6 subclasse, 8 ordens e 13 famílias, 33 espécies e 6 morfo-espécies. A família Triceratiaceae se destacou por apresentar maior diversidade, com 4 gêneros, seguida por Plagiogrammaceae, Lithodesmiaceae e Rhizosoleniaceae, com 2 gêneros cada.

As famílias que apresentaram os maiores números de táxons durante o período de estudo foram: Coscinodiscaceae (11 táxons), Triceratiaceae (8 táxons), Chaetocerotaceae (4 táxons) e Rhizosoleniaceae (3 táxons). As demais famílias representaram 33,3\% sendo estas famílias: Thalassiosiraceae, Lithodesmiaceae, Bellerocheaceae, Plagiogrammaceae, com dois táxons cada e as famílas Skeletonemaceae, Melosiraceae, Aulacodiscaceae, Heliopeltaceae e Hemiaulaceae, apresentando uma única espécie cada (Fig.3).

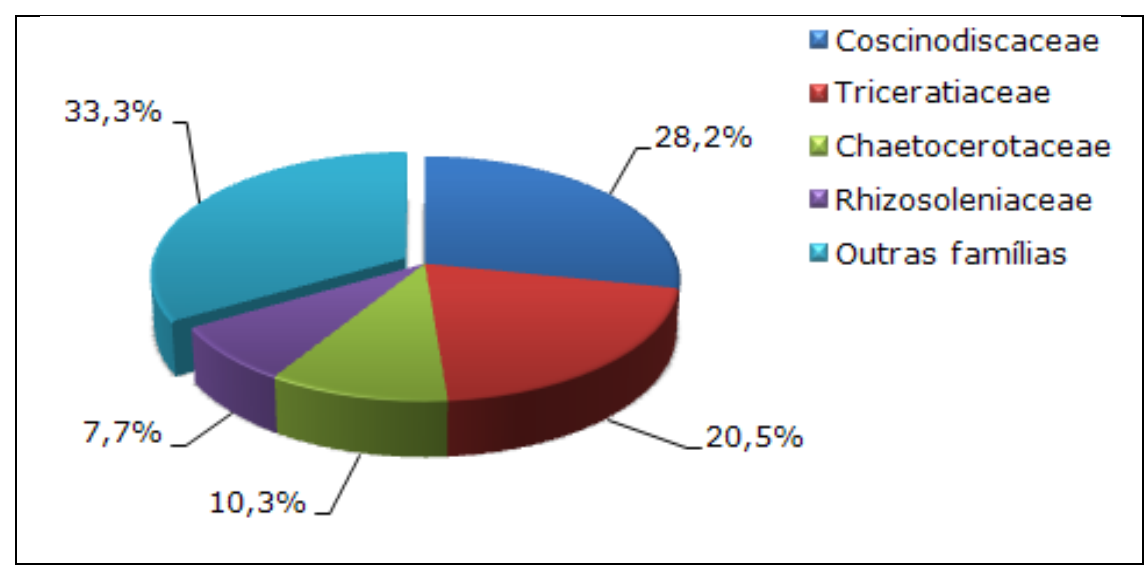

Figure 3 - Representação percentual das famílias mais representativas na praia da Princesa, Ilha de Algodoal.

Durante o presente estudo, as espécies que ocorreram apenas no período seco foram: Aulacodiscus sp., Chaetoceros danicus Cleve, C. decipiens Cleve, C. peruvianus Brightwell, 
Coscinodiscus argus Ehrenberg, C. granii Gough, Plagiogramma sp., Rhizosolenia hebetata Bailey e Thalassiosira sp. As espécies exclusivas do período chuvoso foram: Bellerochea malleus (Brightwell) Van Heurck, Coscinodiscus gigas Ehrenberg, Eupodiscus radiatus Bailey, Hemiaulus sinensis Greville e Lithodesmiun sp. A maioria das espécies exclusivas do período seco são marinhas planctônicas oceânicas ou neríto-oceânicas, enquanto que as do período chuvoso são espécies neríticas.

As espécies marinhas planctônicas representaram 75,8\% das espécies identificadas, enquanto que as marinhas litorais (ticoplanctônicas) representaram 24,2\% dos mesmos. Entre as espécies marinhas planctônicas foram registrados representantes das categorias: marinha planctônica nerítica $(52,4 \%)$, marinha planctônica oceânica $(19,0 \%)$ e marinha planctônica neríto-oceânica $(28,6 \%)$.

Ao longo do período de estudo a maioria das espécies ocorreram de forma esporádica (15 táxons), seguidas pelas muito frequentes (11 táxons), pouco frequentes ( 7 táxons) e frequentes ( 6 táxons). As espécies Coscinodiscus perforatus Ehrenberg, Dimeregramma minor (Gregory) Ralfs, Odontella mobilienses (Bailey) Grunow e Odontella sinensis (Greville) Grunow ocorreram em $100 \%$ das amostras sendo classificadas como muito frequentes. Além disso, Coscinodiscus centralis Ehrenberg (81,2\%), C. concinnus Smith (87,5\%), C. jonesianus (Greville) Ostenfeld $(93,7 \%)$, C. radiatus Ehrenberg $(87,5 \%)$, Melosira nummuloides Agardh $(75,0 \%)$, Odontella aurita (Lyngbye) Agardh $(75,0 \%)$ e Triceratium favus Ehrenberg $(93,7 \%)$ também foram muito frequentes (Fig. 4).

Em termos de abundância relativa, apenas a espécie Coscinodiscus perforatus esteve bem representada em ambos os períodos sazonais, sendo dominante em algumas amostras, com destaque durante os meses chuvosos. Durante o período seco a espécie Dimeregramma minor ocorreu de forma dominante no mês de setembro.

Durante os dois meses do período seco registrou-se um total de 34 espécies. No mês de novembro/2008 Coscinodiscus perforatus variou de dominante (56\%) a abundante (30,3\%), enquanto que Coscinodiscus obscurus Schmidt e Dimeregramma minor foram pouco abundantes (11,2\% e 28,2\%, respectivamente). Em setembro/2009, Dimeregramma minor se destacou como dominante $(56,1 \%)$ e Coscinodiscus perforatus foi pouco abundante $(15,8 \%)$ (Fig. 5). 


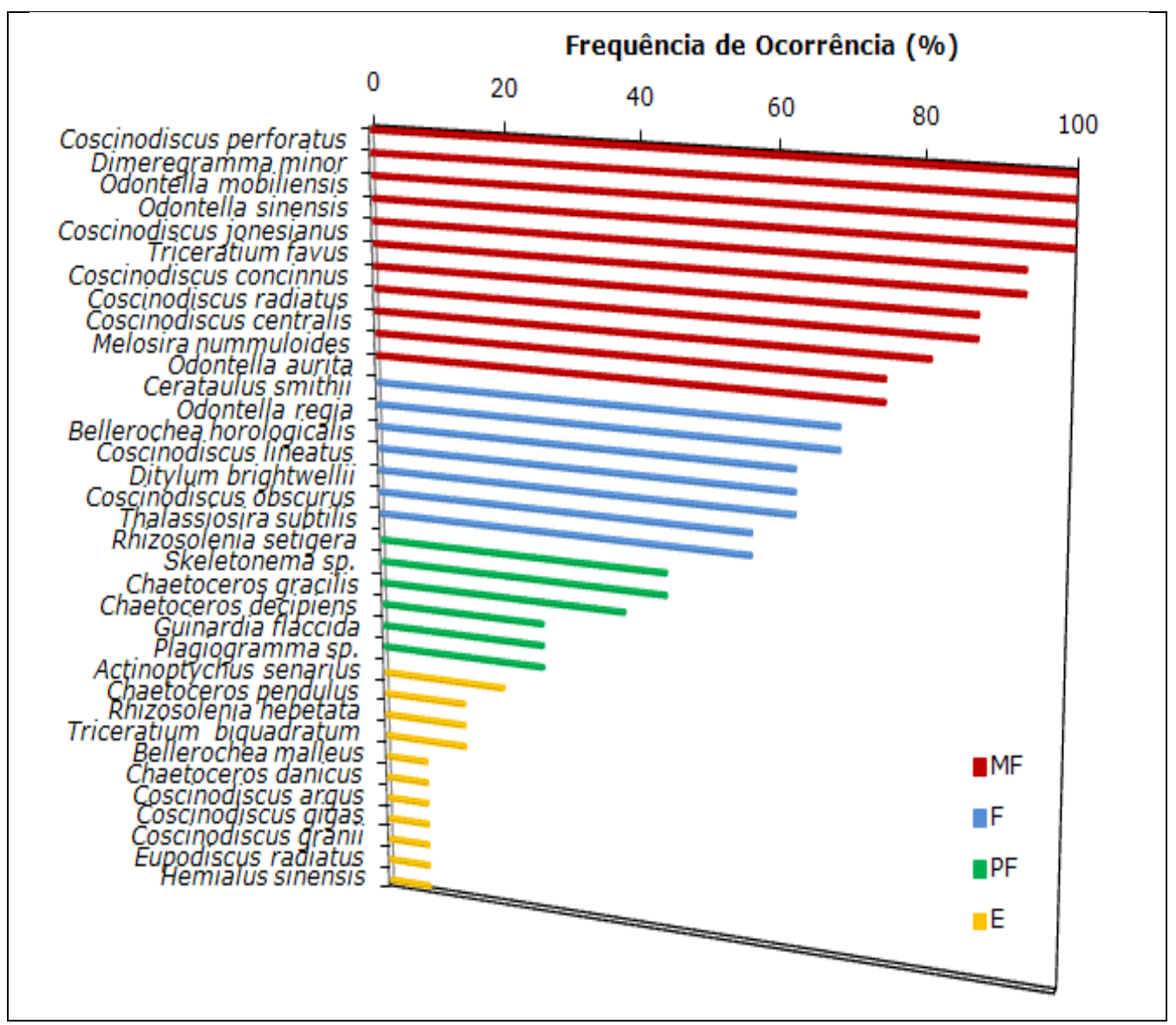

Figure 4 - Frequência de ocorrência das espécies, na praia da Princesa, Ilha de Algodoal, onde E: esporádica; PF: pouco frequente; F: frequente; MF: muito frequente.

Nos meses do período chuvoso foram registrados 29 táxons no total. No mês de março/2009, apenas a espécie Coscinodiscus perforatus foi classificada como dominante $(63,4 \%)$ e a espécie Coscinosdiscus concinnus foi considerada pouco abundante neste mês com 29,2\% de abundância durante a preamar. Em junho/2009, a espécie Coscinodiscus perforatus que foi dominante em ambos regimes de maré, com abundância mais elevada na baixa-mar $(55,6 \%)$. Durante este mês, Coscinodiscus jonesianus foi considerada pouco abundante (preamar) (Fig. 5).

\section{DISCUSSÃO}

Os ambientes costeiros são bastante complexos devido ao seu forte hidrodinamismo, pois sofrem grandes alterações nos seus parâmetros hidrológicos e biológicos (BASTOS; FEITOSA; MUNIZ, 2005; MAGALHÃES et al., 2006; SOUSA et al., 2008). Vários estudos têm demonstrado a influência e importância que a precipitação pluviométrica exerce sobre os parâmetros bióticos e abióticos, principalmente em regiões tropicais (COSTA et al., 2011; MATOS et al., 2012). Nestas áreas, o regime pluviométrico é um dos principais controladores da distribuição, abundância e dinâmica sazonal do fitoplâncton, visto que é diretamente responsável pelas alterações observadas na salinidade local (SASSI, 1991). No entanto, segundo Pritchard (1967), a salinidade não depende apenas da pluviosidade, mas também da descarga de água doce e das marés, podendo ser influenciada também pela circulação dos ventos que atua direta ou indiretamente sobre a superfície da água.

Acentuadas variações de salinidade foram registradas ao longo do presente estudo, sendo observada uma relação de dependência entre a salinidade e os índices pluviométricos locais. No primeiro semestre de 2009 ocorreram os maiores índices pluviométricos (evento de La Niña), com destaque para o mês de março/09, enquanto que no segundo semestre foram registradas poucas chuvas (INMET, 2009) e os mais elevados valores de salinidade (redução da pluviosidade e aumento da evaporação). Este fato demonstra claramente a importância da 
sazonalidade das chuvas sobre as variações dos parâmetros hidrológicos, e em especial da salinidade, na região costeira amazônica, como observado por Pereira et al. (2012), em estudos realizados na ilha de Algodoal.

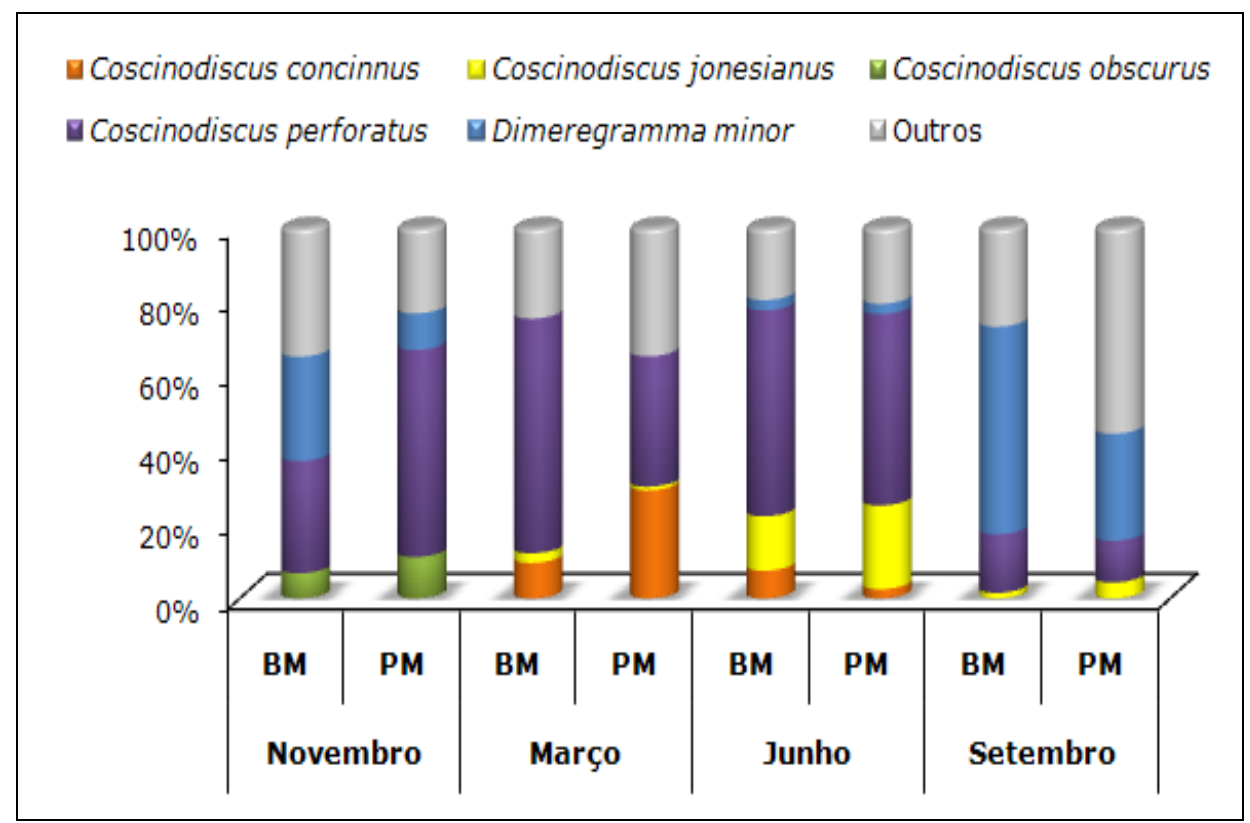

Figure 5 - Abundância relativa das diatomáceas cêntricas na praia da Princesa, Ilha de Algodoal, durante o período de estudo.

A temperatura é um importante parâmetro nos ecossistemas aquáticos, contudo, em áreas tropicais sua relevância é minimizada devido a sua pequena amplitude térmica e as gradativas alterações sofridas pela mesma ao longo do ciclo sazonal. A temperatura da água na praia da Princesa apresentou pequena oscilação anual, o que é característico de regiões tropicais e equatoriais (BASTOS; FEITOSA; MUNIZ, 2005; COSTA et al. 2011; MATOS et al. 2011), estando relacionada, principalmente, com as variações nas taxas de precipitação e à intensidade luminosa, não sendo, portanto, um fator determinante da dinâmica populacional das comunidades planctônicas locais (MAGALHÃES et al., 2006; MATOS et al., 2012).

Os teores de oxigênio dissolvido na água constituem outro importante parâmetro nos ecossistemas aquáticos em função de seu papel nos processos bioquímicos e biológicos tais como respiração, oxidação da matéria orgânica, e processos metabólicos celulares indispensáveis para a vida vegetal e animal (NORIEGA et al., 2005). Na área estudada verificou-se que as maiores concentrações de oxigênio dissolvido foram registradas durante o mês de setembro/2009. Provavelmente, este padrão esteve relacionado à forte hidrodinâmica local, bem como as trocas oceano/atmosfera ocasionadas principalmente pela ação dos ventos, correntes de marés e ondas, proporcionando uma maior oxigenação da água (SILVA et al., 2009; PEREIRA et al., 2012).

$\mathrm{O} \mathrm{pH}$ manteve-se alcalino durante todo o período de estudo em ambos os regimes de maré, como verificado nos trabalhos realizados no nordeste do Brasil, como o de Feitosa, Nascimento e Costa (1999) na Bacia do Pina (PE), Losada, Feitosa e Correia-Lins (2000) nos rios Ilhetas e Mamucaba na Baía de Tamandaré (PE) e Costa e Macêdo (1987/89) no rio Timbó $(\mathrm{PE})$. De forma geral, valores elevados de $\mathrm{pH}$ em zonas costeiras são comumente associados ao efeito tampão da água marinha (SCHMIEGELOW, 2004) e previamente descritos para ambientes costeiros e estuarinos amazônicos (COSTA; PEREIRA; COSTA, 2008; SOUSA et al., 2009).

Durante o período de estudo, os valores de turbidez foram bastante elevados principalmente no mês de setembro/2009, possivelmente em decorrência da elevada hidrodinâmica local observada durante a maré equinocial de sizígia. No mês de junho/2009, também foram registradas águas mais turvas, provavelmente em decorrência da grande descarga de águas continentais, uma vez que, a elevada vazão dos rios e estuários amazônicos (que aportam uma grande quantidade de sedimentos, nutrientes e matéria orgânica) e a 
elevada hidrodinâmica local são fatores que contribuem para que as águas costeiras amazônicas sejam de elevada turbidez (GEYER et al. 1996; PEREIRA et al. 2009; COSTA et al., 2011).

Em regiões tropicais, dependendo da variabilidade dos parâmetros hidrológicos de cada ambiente, as maiores concentrações de clorofila a podem ocorrer no período chuvoso ou de estiagem. O principal fator condicionante para que isso ocorra, é a precipitação, uma vez que ela contribui para o enriquecimento de sais nutrientes na água, ao mesmo tempo em que aumenta a concentração de material em suspensão, levando ao aumento da biomassa ou limitando a camada fótica e reduzindo o desenvolvimento do fitoplâncton (LOSADA; FEITOSA; CORREIA-LINS, 2000). Na praia da Princesa, os teores de clorofila a foram consideravelmente mais elevados durante o mês de março/2009, sugerindo uma maior disponibilidade de nutrientes dissolvidos na água (ambiente eutrófico) e possibilitando o desenvolvimento de uma proliferação ("bloom") pontual de Coscinodiscus perforatus, caracterizada por uma elevada biomassa algal. Isto sugere que o aumento da turbidez no início do período chuvoso não limita, ao menos no mês estudado, o crescimento fitoplanctônico na área estudada.

As diatomáceas constituem um dos principais grupos microalgais em águas costeiras (DEVASSY; GOES, 1988; ESTRADA et al., 1999; PUIGSERVER; RAMON; MOYA, 2002) e representam os principais produtores primários da zona de arrebentação de praias arenosas (BROWN; MCLACHLAN, 1990; TALBOT; BATE; CAMPBELL, 1990). De acordo com Parsons, Takahashi e Hargrave (1984), as diatomáceas são registradas tanto em regiões costeiras quanto em oceânicas e sua riqueza taxonômica tem sido demonstrada para a maioria dos ambientes costeiros amazônicos, como observado nos trabalhos realizados por Sousa et al. (2008), na Ilha Canela (PA), Costa et al. (2011) na praia de Ajuruteua e Matos et al. (2011) no estuário do Caeté (PA).

As diatomáceas cêntricas são as principais constituintes do microfitoplâncton de águas costeiras. No presente estudo, o gênero Coscinodiscus Ehrenberg foi o mais numeroso taxonomicamente, fato este previamente observado no estuário do rio Caeté por Matos et al. (2011). Várias espécies do gênero Coscinodiscus têm sido referidas como muito frequentes e consideradas características de ambientes costeiros do nordeste paraense, tais como a praia de Ajuruteua (SANTANA; PAIVA; MELO, 2005). Os representantes deste gênero, apesar de terem sido registrados em ambos os períodos sazonais, foram mais representativos durante o período chuvoso, principalmente a espécie Coscinodiscus perforatus, que foi abundante na maioria das amostras do presente estudo.

A espécie marinha litoral (ticoplanctônica) Dimeregramma minor, observada em comunidades bentônicas sobre a superfície de sedimentos de mangue (HASSAN; ESPINOSA; ISLÃ, 2006) e sedimentos arenosos (COOK; ROY, 2006), foi muito representativa durante 0 período seco, uma vez que esta espécie é polihalóbia estando mais adaptada às condições de altas salinidades. De acordo com Losada, Feitosa e Correia-Lins (2000) isto pode ocorrer devido a maior homogeneização da coluna d"água e ressuspensão dos sedimentos, nutrientes e organismos bentônicos, tais como da referida espécie, alterando a estrutura fitoplanctônica local como sugerido por Sousa et al. (2009) e Matos et al. (2011).

No presente estudo, o maior número de táxons foi registrado nos meses do período seco como frequentemente observado em outros ambientes costeiros amazônicos (SOUSA et al., 2008). Este fato se deve, possivelmente, as condições de luminosidade observadas neste período (LACERDA et al., 2004) e pelas características das diatomáceas de suportar grandes variações de salinidade (PATRICK, 1967).

As condições hidrológicas características de zona de arrebentação proporcionaram a dominância de espécies marinhas planctônicas neríticas, além da presença de espécies marinhas litorais e outras caracteristicamente oceânicas. Resultados similares têm sido observados por diversos autores em ambientes costeiros do Nordeste paraense, tais como o estuário do rio Caeté (MATOS et al., 2011), a Ilha Canela (SOUSA et al., 2008) e anteriormente na Ilha de Algodoal (MATOS et al., 2012).

Segundo Frenguelli (1928), é difícil estabelecer limites exatos no que se refere a uma separação nítida entre formas oceânicas e formas planctônicas neríticas, visto que há um 
grande número de espécies que podem ser encontradas em mar aberto, bem como próximo a costa. Além disso, inúmeras espécies são capazes de adaptar-se a todas as condições de temperatura e salinidade das águas marinhas, tais como as diatomáceas que predominam ambientes costeiros devido às suas características de eurialinidade (PATRICK, 1967).

A estrutura das populações do fitoplâncton encontra-se diretamente relacionada às características físicas e químicas da água conjuntamente com outros fatores ambientais, os quais, agindo em conjunto ou isoladamente, condicionam o estabelecimento de populações adaptadas a estas variações (PHLIPS; BADYLAK; GROSSKOPF, 2002). Na praia da Princesa, mudanças temporais na distribuição e abundância das diatomáceas cêntricas foram reguladas principalmente pela precipitação e pela ação dos ventos e correntes de maré, as quais favorecem a homogeneização da coluna d'água, o que permite a presença de espécies marinhas planctônicas e marinhas litorais (ticoplanctônicas), que caracterizaram a estrutura do fitoplâncton local.

\section{CONCLUSÃO}

De forma geral, a sazonalidade local exerceu uma forte influência sobre as variáveis ambientais, e em especial sobre a salinidade, a qual parece constituir o principal fator controlador da ocorrência e da abundância dos organismos microfitoplanctônicos na praia da Princesa. Possivelmente, a ação dos ventos e correntes de maré, favoreceram também a homogeneização da coluna d'água, permitindo a contribuição de diferentes espécies em distintos períodos sazonais. A espécie marinha litoral (ticoplanctônica) Dimeregramma minor foi muito representativa durante o período seco, estando bem adaptada às condições de altas salinidades. No entanto, durante o período chuvoso a espécie que mais se destacou foi Coscinodiscus perforatus, comumente observada em ambientes costeiros amazônicos, apesar de ter sido observada em ambos os períodos sazonais.

\section{AGRADECIMENTOS}

A FAPESPA pelo financiamento dos projetos Universal (116/2008) e PROSGRAD $(070 / 2008)$, os quais o presente estudo esteve vinculado. Os autores agradecem ao CNPq pela concessão da bolsa e mestrado à primeira autora, pelo suporte financeiro oferecido através dos projetos CT-Hidro casadinho (\# 552126/2005-5) e Taxonomia (\# 563967/05-6), bem como pelas bolsas de pesquisa \# 308379/2011-0 e \# 306061/2011-2, concedidas ao Dr. Rauquirio Marinho da Costa e a Dra. Luci Cajueiro Carneiro Pereira, respectivamente.

\section{REFERÊNCIAS}

BASTOS, M. N. C. Caracterização das formações vegetais da restinga da Princesa, Ilha de Algodoal-PA. Belém, 1996. 261f. Tese (Doutorado em Ciências Biológicas) - Universidade Federal do Pará.

BASTOS, R. B.; FEITOSA, F. A. N.; MUNIZ, K. Variabilidade espaço-temporal da biomassa fitoplanctônica e hidrologia no estuário do Rio Una (Pernambuco - Brasil). Tropical

Oceanography, v. 33, n. 1, p. 1-18, 2005.

BELL, P. R.; HEMSLEY, A. R. Green Plants: Their Origin and Diversity. New York: Cambridge University Press, 2000. 349p.

BROWN, A. C.; MCLACHLAN, A. Beach and surf-zone flora. In: BROWN, A. C.; MCLACHLAN, A. (Ed.). Ecology of Sandy Shores. Netherlands: Elsevier Science, 1990. p. 41-50.

CONOVER, W. O. J. Practical nonparametric statistics. New York: John Wiley, 1971. 302p.

COOK, P. M. L.; ROY, H. Advective relief of $\mathrm{CO} 2$ limitation in microphytobenthos in highly productive sandy sediments. Limnology and Oceanography, v. 51, n. 4, p. 1594-1601, 2006.

COSTA, A. J. G.; COSTA, K. G.; PEREIRA, L. C. C.; SAMPAIO, M. I. ; COSTA, R. M. da. Dynamics of hydrological variables and the fish larva community in an Amazonian estuary of northern

Brazil. Journal of Coastal Research, v. SI 64, p. 1960-1964, 2011. 
COSTA, K. G.; PEREIRA, L. C. C.; COSTA, R. M. da. Short and long-term temporal variation of the zooplankton in a tropical estuary (Amazon region, Brazil). Boletim do Museu Paraense Emílio Goeldi, Série Ciências Naturais, v. 3, n. 2, p. 127-141, 2008.

COSTA, K. M. P.; MACEDO, S. J. Estudo hidrológico do Rio Timbó (Pernambuco-Brasil). Trabalhos Oceanográficos da Universidade Federal de Pernambuco, v. 2, p. 7-34, $1987 / 1989$.

COSTA, V. B.; SOUSA, E. B.; PINHeIRO, S. C. C.; PEREIRA, L. C. C.; COSTA, R. M. da. Effects of a high energy coastal environment on the structure and dynamics of phytoplankton communities (Brazilian Amazon littoral). Journal of Coastal Research, v. SI 64, p. 354-358, 2011.

DEVASSY, V. P.; GOES, J. I. Phytoplankton Community Structure and Succession in a Tropical Estuarine Complex (Central West Coast of India). Estuarine, Coastal and Shelf Science, v. 27, p. 671-685, 1988.

ESTRADA, M.; VARELA, L. A.; SALAT, J.; CRUZADO, A.; ARIAS, E. Spatio-temporal variability of the winter phytoplankton distribution across the Catalan and North Balearic fronts (NW Mediterranean). Journal of Plankton Research, v. 21, n. 1, p. 1-20, 1999.

FEITOSA, F. A. N.; NASCIMENTO, F. C. R.; COSTA, K. M. P. Distribuição espacial e temporal da biomassa fitoplanctônica relacionada com parâmetros hidrológicos na Bacia do Pina (Recife-PE). Trabalhos Oceanográficos da Universidade Federal de Pernambuco, v. 27, n. 2, p. 1-13, 1999.

FRENGUELLI, J. Diatomeas del Oceano Atlantico. Anais do Museo Nacional Historia Naturale Bernardino Rivadavia, Buenos Aires, v. 34, p. 497-572, 1928.

GEYER, W. R.; BEARDSLEY, R. C.; LENTZ, S. J.; CANDELA, J.; LIMEBURNER, R.; JOHNS, W. E.; CASTRO, B. M.; SOARES, I. D. Physical oceanography of the Amazon shelf. Continental Shelf Research, v. 16, n. 5-6, p. 575-616, 1996.

HASSAN, G.S.; ESPINOSA, M.A.; ISLÃ, F.I. Modern diatom assemblages in surface sediments from estuarine systems in the southeastern Buenos Aires Province, Argentina. Journal of Paleolimnology, v. 35, p. 39-53, 2006.

INMET. Normas climatológicas. Instituto Nacional de Meteorologia, Brasilia/DF, 2009. Disponível em http://www.inmet.gov.br. Acessado em 30 de dezembro de 2009.

KRAUSE, G.; GLASER, M. Co-evolving geomorphological and socio-economic dynamics in a coastal fishing village of the Bragança Region (Pará, North Brazil). Ocean \& Coastal Management, v. 46, p. 859-874, 2003.

LACERDA, S. R.; KOENING, M. L.; NEUMANN-LEITÃO, S.; FLORES-MONTES, M. J. Phytoplankton nyctemeral variationat a tropical riverestuary (Itamaracá-Pernambuco-Brasil). Brazilian Journal Biology, v. 64, n. 1, p. 81-94, 2004.

LOSADA, A. P. M.; FEITOSA, F. A. N.; CORREIA-LINS, I. Variação sazonal e espacial da biomassa fitoplanctônica nos estuários dos rios Ilhetas e Mamucaba (Tamandaré-PE) relacionada com parâmetros hidrológicos. Trabalhos Oceanográficos da Universidade Federal de Pernambuco, v. 28, n. 2, p. 117-138, 2000.

MAGALhãES, A.; COSTA, R. M.; LIANG, T. H.; PEREIRA, L. C. C.; RIBEIRO, M. J. S. Spatial and temporal distribution in density and biomass of two Pseudodiaptomus species

(Copepoda: Calanoida) in the Caeté River Estuary (Amazon region - North of Brazil). Brazilian Journal of Biology, v. 66, n. 2A, p. 421-430, 2006.

MARGULIS, L.; SCHWARTZ, K. V. Five Kingdoms: An illustrated Guide to the Phyla of Life on Earth. New York: WH. Freemann and Company, 1998. 497p.

MARTORANO, L. G.; PERREIRA, L. C.; CÉSAR, E. G. M.; PEREIRA, I. C. B. Estudos climáticos do Estado do Pará, classificação climática (Köppen) e deficiência hídrica (Thornthwhite Mather). Belém: SUDAM/EMBRAPA, SNLCS, 1993: 53p. 
MASCARENHAS, A. L. S. Análise Ambiental da ilha de Algodoal-Maiandeua/PA. Fortaleza, 2006. 133f. Dissertação (Mestrado em Geografia) - Departamento de Geografia, Universidade Federal do Ceará.

MATOS, J. B.; SODRÉ, D. K. L.; COSTA, K. G.; PEREIRA, L. C. C.; COSTA, R. M. da. Spatial and temporal variation in the composition and biomass of phytoplankton in an Amazonian estuary. Journal of Coastal Research, v. SI 64, p. 1525-1529, 2011.

MATOS, J. B.; SILVA, N. I. S.; PEREIRA, L. C. C.;COSTA, R. M. da. Caracterização qualiquantitativa do fitoplâncton da zona de arrebentação de uma praia amazônica. Acta Botanica Brasilica, v. 26, n. 4, p. 979-990, 2012.

MATTEUCCI, S. D.; COLMA, A. La metodología para el estudio de la vegetación.

Washington: Secretaria General de la Organización de los Estados Americanos, 1982. 168p.

MONTEIRO, M. C.; PEREIRA, L. C. C.; OLIVEIRA, S. M. O. de. Morphodynamic changes of a macrotidal sand beach in the Brazilian Amazon coast (Ajuruteua-Pará). Journal of Coastal Research, v. SI 56, p. 103-107, 2009.

MORAES, B. C.; COSTA, J. M. N.; COSTA, A. C. L.; COSTA, M. H. Variação espacial e temporal da precipitação no Estado do Pará. Acta Amazonica, v. 35, n. 2, p. 207-214, 2005.

MOREIRA FILHO, H.; VALENTE-MOREIRA, I. M.; SOUZA-MOSIMANN, R. M.; CUNHA, J. A. Avaliação florística e ecológica das diatomáceas (Chrysophyta, Bacillariophyceae) marinhas e estuarinas nos Estados do Paraná, Santa Catarina e Rio Grande do Sul. Estudos de Biologia, v. 25 , p. $5-48,1990$.

NORIEGA, C. D.; COSTA, K. M. P. da; FEITOSA, F. A. N.; FLORES-MONTES, M. J.; GREGO, C. K. S.; SOARES, G. S. S.; SILVA, H. P. da. Distribuição espacial da biomassa fitoplanctônica e sua relação com os sais nutrientes, no sistema estuarino de barra das Jangadas (Pernambuco Brasil). Arquivos de Ciências do Mar, v. 38, p. 5-18, 2005.

PARSONS, T. R.; STRICKLAND, J. D. H. Discussion of spectrophotometric determination of marine plankton pigments, with revised equations of as artaining clorophyllaanol carotenoids.

Journal of Marine Research, v. 21, n. 3, p. 155-163, 1963.

PARSONS, T. R.; TAKAHASHI, M.; HARGRAVE, B. (Ed.). Biological Oceanographic Processes. $3^{a}$ ed Oxford: Pergamon Press, 1984. 330 p.

PATRICK, R. Diatoms communities in estuaries. In: LAUFF, G. H. (Ed). Estuaries. Washington: American Association for the Advance of Science, 1967. 311-315p.

PEREIRA, L. C. C.; MENDES, C. M.; MONTEIRO, M. C.; ASP, N. E. Morphological and sedimentological changes in a macrotidal sand beach in the Amazon Littoral (Vila dos Pescadores, Pará, Brazil). Journal of Coastal Research, v. SI 56, p. 113-117, 2009.

PEREIRA, L. C. C.; SILVA, N. I. S.; COSTA, R. M.; ASP, N. E.; COSTA, K. G.; VILA-CONCEJO, A. Seasonal changes in oceanographic processes at an equatorial macrotidal beach in northern Brazil. Continental Shelf Research, v. 43, p. 95-106, 2012.

PHLIPS, E. J.; BADYLAK, S.; GROSSKOPF, T. Factors affecting the abundance of phytoplankton in a restricted subtropical lagoon, the Indian River Lagoon, Florida, USA. Estuarine, Coastal and Shelf Science, v. 55, p. 385-402, 2002.

PRITCHARD, D. W. What is an estuary: Physical viewpoint. In: LAUFF, G. H. Estuaries. Washington: American Association for the Advance of Science, 1967. 3-5p.

PUIGSERVER, M.; RAMON, G.; MOYA, G. Spatial and temporal distribution of phytoplankton in a Mediterranean estuarine channel system. Journal of Coastal Research, v. 18, n. 1, p. 39-51, 2002.

REYNOLDS, C. S. Dynamics, selection and composition of phytoplankton in relation to vertical structure in lakes. Archieve Hydrobiology, v. 35, p. 13-31, 1992.

ROUND, F. E.; CRAWFORD, R. M.; MANN, D. G. The Diatoms, Biology Morphology of the

Genera. Cambridge: Cambridge University, 1990. 1747p. 
SANTANA, D. S.; PAIVA, R. S.; MELO, N. F. A. C. Diatomácias cêntricas da região da praia de Ajuruteua (Bragança, Pará). Boletim do Museu Paraense Emílio Goeldi, Série Ciências Naturais, v. 1, n. 3, p. 109-116, 2005.

SASSI, R. Phytoplankton and environmental factor in the Paraíba do Norte river estuary, Northeastern Brazil: composition, distribution and quantitative remarks. Boletim do Instituto Oceanográfico, v. 39, n. 2, p. 93-115, 1991.

SCHMIEGELOW, J. M. M. O planeta azul: uma introdução às ciências marinhas. Rio de Janeiro: Interciência, 2004. 202p.

SILVA, I. R. da; PEREIRA, L. C. C.; GUIMARÃES, D. O.; TRINDADE, W. N.; ASP, N. E.; COSTA, R. M. da. Environmental status of urban beaches in São Luís (Amazon Coast, Brazil). Journal of Coastal Research, v. SI 56, p. 1301-1305, 2009.

SILVA-CUNHA, M. G.; ESKINAZI-LEÇA, E. Catálogo das Diatomáceas (Bacillariophyceae) da Plataforma continental de Pernambuco. Recife: SUDENE/UFPE, 1990. 318p.

SILVA, N. I. S.; PEREIRA, L. C. C.; GORAYEB, A.; VILA-CONCEJO, A.; SOUSA, R. C.; ASP, N. E.; COSTA, R. M. da. Natural and social conditions of Princesa, a macrotidal sandy beach on the Amazon Coast of Brazil. Journal of Coastal Research, v. SI 64, p.1979-1983, 2011.

SOKAL, R. R.; ROHLF, F. J. Biometry: The principles and practice of numerical classification in biological research. San Francisco, California: W. H. Freeman, 1969. 776p.

SOUSA, E. B.; COSTA, V. B.; PEREIRA, L. C. C.; COSTA, R. M. da. Microfitoplâncton de águas costeiras amazônicas: Ilha Canela (Bragança, PA, Brasil). Acta Botanica Brasilica, v. 22, n. 3, p. 626-636, 2008.

SOUSA, E. B.; COSTA, V. B.; PEREIRA, L. C. C.; COSTA, R. M. da. Variação temporal do fitoplâncton e dos parâmetros hidrológicos da zona de arrebentação da Ilha Canela (Bragança, PA, Brasil). Acta Botanica Brasilica, v. 23, n. 4, p. 1084-1095, 2009.

SOUSA, R. C. de; PEREIRA, L. C. C.; SILVA, N. I. S.; OliVEIRA, S. M.; PINTO, K. S. T.; COSTA, R. M. da. Recreational carrying capacity of three Amazon macrotidal beaches during the peak vacation season. Journal of Coastal Research, v. SI 64, p.1292-1296, 2011.

STRICKLAND, J. D. H.; PARSONS, T. R. A Practical Handbook of Seawater Analysis. Bulletin Fisheries Research Board of Canada, v. 167, p. 1-311, 1968.

SURTHERS, I. M.; RISSIK, D. (Eds). Plankton: a guide to their ecology and monitoring for water quality. Collingwood: Csiro Publishing, 2008. 256p.

TALBOT, M. M. B.; BATE, G. C.; CAMPBELL, E. E. A review of the ecology of surf zone diatoms, with special reference to Anaulus australis. Oceanography and Marine Biology. Na Annual Review, v. 28, p. 155-175, 1990.

TOMAS, C. R. Identifying marine phytoplankton. San Diego: Academic Press, 1997. 858p.

VALENTE-MOREIRA, I. M.; MOREIRA FILHO, H.; CUNHA, J. A. Diatomáceas (Chrysophyta, Bacillariophyceae) em biótopo de manguezal do rio Perequê, em Pontal do Sul, Paranaguá, Estado do Paraná, Brasil. Acta Biológica Paranaense, v. 23, n. 1, 2, 3, 4, p. 55-72, 1994.

VALIELA, I. Marine ecological processes. $2^{\circ}$ Ed. New York: Springer-Verlag. 1995. 686p.

VAN DEN HOEK, C.; MANNS, D. G.; JAHNS, H. M. Algae, an introduction to phycology. New York: Cambridge University Press, 1995. 623p.

WOOD, E. J. F. A phytoplankton study of the Amazon region. Bulletin of Marine Science, $v$. 16, p. 102-123, 1968.

ZAR, J. H. Bioestatistical analysis. 4a ed. New Jersey: Prentice Hall. 1999. 931p. 